\title{
Paroxysmal Nocturnal Haemoglobinuria (PNH): Rare Clinical Presentation of this Rare Disease
}

\section{Authors \\ Dr Gangadhar ${ }^{1}$, Dr Sahil Arora ${ }^{1}$, Dr Prabal Rajvanshi ${ }^{2}$, Dr Swathi $T^{1}$ Dr Jogendra Singh ${ }^{1}$}

${ }^{1}$ Senior Resident, Department of Medicine, Vardhman Mahavir Medical College and Safdarjung Hospital, New Delhi-110029

${ }^{2}$ Professor and Consultant, Department of Medicine, Vardhman Mahavir Medical College and Safdarjung

Hospital, New Delhi-110029

Corresponding Author

\section{Dr Gangadhar}

07 Mithuna Block, Police Quarters, Tajsultanpur. Gulbarga, Karnataka- 585104, India

Email: gangadharbuduga@gmail.com

\section{Abstract}

Paroxysmal Nocturnal Haemoglobinuria is a rare haematological disease causing acquired haemolytic anaemia. The clinical spectrum of the disease starts from anaemia (microcytic to macrocytic) to pancytopenia and aplastic anaemia. The disease may present at any age group but the most common prevalence is in the third and fourth decade. This disease is not diagnosed commonly as the classical haematuria is not present in majority. We present the case of 15-year-old boy who presented with skin rash all over body and weakness, which on investigation was diagnosed as case of PNH. Skin manifestation of PNH are very uncommon and rarely reported in literature. A high index of suspicion is necessary for the diagnosis.

Keywords: paroxysmal nocturnal haemoglobinuria, ecchymosis, haemolysis, haemoglobinuria, CD55 and CD59.

\section{Introduction}

Paroxysmal nocturnal haemoglobinuria $(\mathrm{PNH})$ also known as Marchiafava-Micheli syndrome was reported as early as 1793 by Charles steward. The essential features of this disease were described by Paul Strubing in 1882. Its prevalence was estimated to be approximately 2 per million with equal frequency in both males and females. $\mathrm{PNH}$ is an acquired hematopoietic stem cell disorder. The disease diagnosis is usually attributed to its clinical features like haemoglobinuria, haemolytic anaemia, venous thrombosis and marrow failure.
Haemoglobinuria has been traditionally been the defining feature of $\mathrm{PNH}$. Haemoglobinuria is attributed to intravascular haemolysis, yet detected only in few patients. In addition to haemolysis, individuals with $\mathrm{PNH}$ are also susceptible to developing repeated, potentially life-threatening blood clots (thromboses). Affected individuals also have some degree of underlying bone marrow dysfunction and rarely terminating in acute myeloid leukaemia. The specific symptoms of PNH vary greatly from one person to another and affected 
individuals usually do not exhibit all of the symptoms associated with the disorder.

\section{Case Report}

A 15-year-old boy presented in outpatient department of Safdarjung hospital, which is tertiary care hospital in new Delhi, with complaints of pink to brown rashes all over the body for 13 days. Those lesions were looked like a healed lesions of chicken pox. Patient denied of any fever in past these days of illness. Initially noted on the face, which are then involved the whole body and not associated with itching or burning sensation. On enquiring the patients father he gives a similar history of these kind of rashes all over body in past recent rashes were seen 5 months ago, which was also associated with low grade fever for 2-3 days and which got fades in few days with treatment from local hospital. Also gives history of yellowish discolouration of eyes one year ago. This is the fourth time of similar rashes all over body.

On examination patient was looking pallor, afebrile to touch. Vitals were normal with $\mathrm{PR}=76 / \mathrm{min}$, BP $=124 / 64 \mathrm{mmhg}$ of mercury. There were multiple well defined erythematous macules present all over body, non-tender on touching them and looks to be ecchymosis patches(figure1\&2). Per abdomen examination showed spleen enlargement about $3 \mathrm{cms}$ from right costal margin towards umbilicus, rest of systems examination were normal. On investigation his complete blood picture $\mathrm{Hb}$ was $8.3 \mathrm{~g} / \mathrm{dl}$, total leukocyte counts $3100 / \mathrm{dl}$, platelet count $65000 / \mathrm{mm}^{3}$. Haematocrit was $39 \%$, MCV 73 fl. Suggestive of pancytopenia, corrected reticulocyte count was $0.9 \%$ which is lower than normal suggestive of defect in bone marrow RBC synthesis. Peripheral picture showed normocytic to normochromic anaemia with some abnormal RBC as bite cells. LFT suggestive increased indirect bilirubin with total bilirubin was 2.8 , indirect was 2.0. SGOT and SGPT were normal. Rest of the basic blood reports were normal. LDH was in normal range, haptoglobulin was undetectable. Indirect coombs test was negative. ANA was negative. HIV /Hepatitis $\mathrm{B}$ and $\mathrm{C}$ were negative.
USG abdomen showed moderate splenomegaly, and $\mathrm{x}$ ray chest, $2 \mathrm{~d}$ echo were normal. Bone marrow showed hypo-cellular picture with fat space $>30 \%$. Myeloid erythroid ratio was 3:1 erythroid hyperplasia with normoblastic reaction noted. megakaryocytes are markedly reduced

Skin biopsy was taken and it showed as subcutaneous arteriolar occlusion (figure3). Finally, flow cytometry test showed that RBC and some granulocytes were deficient CD59 and CD55 on their surface. We could not do acidified serum (Ham) test due to non-availability of this test in our hospital setup. We took second opinion from the haematologist of our institute and labelled this as a case of PNH with pancytopenia.

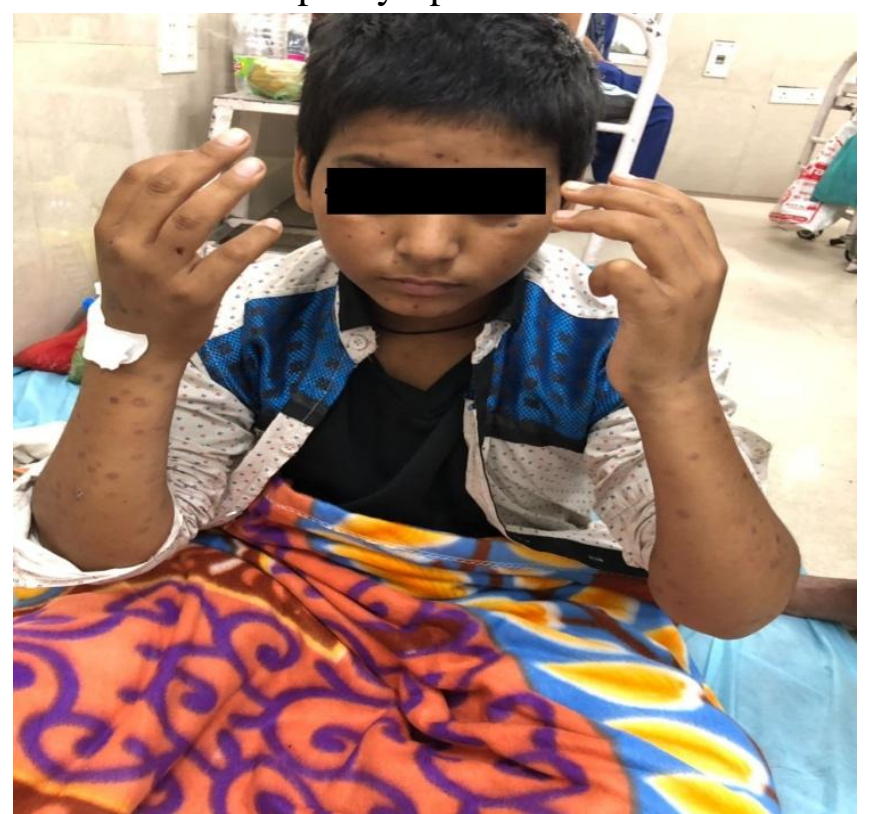

Figure 1: showing skin lesions over body (upper limbs and face)

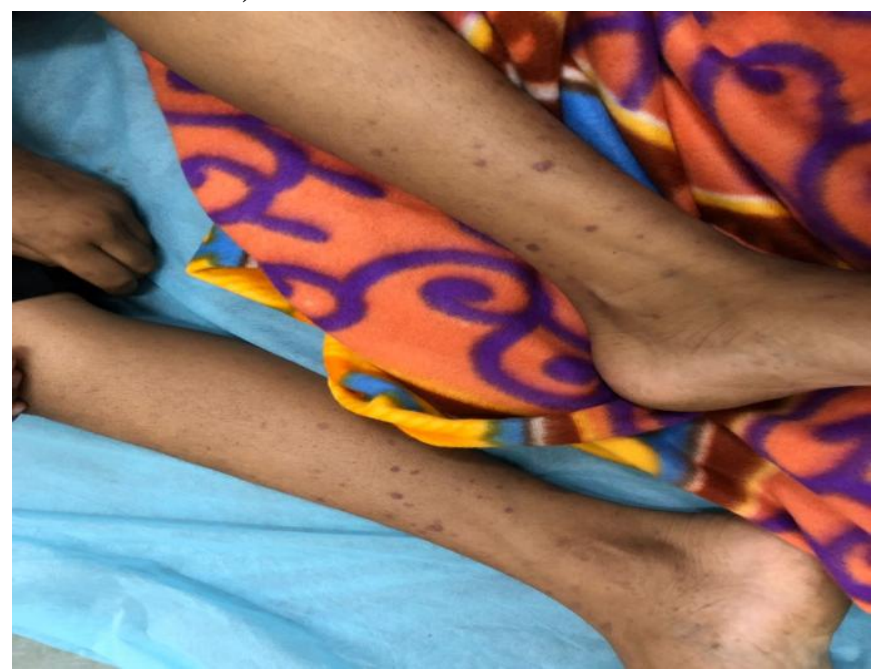

Figure 2: showing same skin lesions over lower limbs. 
In hospital course patient developed epistaxis and gum bleed on $3^{\text {rd }}$ day of admission, this time platelet count was on very lower side, $23000 / \mathrm{mm}^{3}$.we transfused 6 pack of platelet rich plasma to him. epistaxis stopped after transfusion of platelets.

Then we started him on eculizumab intravenously every 14 days. which binds the complement component C5 near the site and prevent haemolysis further. Folic acid tablets given daily. And practiced pure aseptic precaution with him as his total leucocyte count were on lower side, but we dint started any prophylaxis of antibiotics as he dints developed fever and neutrophil were $800 / \mathrm{mm}^{3}$. After 3 transfusions of eculizumab patient blood parameter showed improvement, then we discharged him on ferrous sulphate and folic acid tablets. And planning for the allogenic bone marrow transplant from haematology department of our hospital.

\section{Discussion}

As a common knowledge of us PNH presents as one of the manifestations of its triad; haemolysis, aplasia and thrombosis. But it was different in our case presentation.Leg ulcers and purpura similar to the manifestation of thrombotic thrombocytopenic purpura (TTP), disseminated intravascular coagulation, and Henoch-Schönlein purpura do occur in PNH as we look back in some of case reports. only five cases of $\mathrm{PNH}$-associated cutaneous thrombosis have been reported till now ${ }^{(1-}$ 5). Thrombosis is one of the most immediately lifethreatening complications of $\mathrm{PNH}$ and yet one of the least understood in its pathogenesis. It could be that deficiency of CD59 on the PNH platelet causes inappropriate platelet activation; however, other mechanisms are possible. But it involves commonly large veins of abdominal organs as hepatic vein thrombosis (budd Chiari syndrome). But in our case it was different, it involved the small subcutaneous arterioles which got resolved without any intervention.

Haemolysis in PNH is mainly intravascular and is due to an intrinsic abnormality of the red cell, which makes it exquisitely sensitive to activated $\mathrm{C}$, whether it is activated through the alternative pathway or through an antigen-antibody reaction. The former mechanism is mainly responsible for chronic haemolysis in $\mathrm{PNH}$; Hyper susceptibility to $\mathrm{C}$ is due to deficiency of several protective membrane proteins, of which CD59 is the most important, because it hinders the insertion into the membrane of C9 polymers. The shortage of GPI is due in turn to a mutation in an X-linked gene, called $P I G-A$, required for an early step in GPI biosynthesis.

Supportive care of PNH includes the blood product transfusion only when absolute indication is present as in our case it was epistaxis and gum bleeding with very low platelet count. Unnecessary exposure of the patient is avoided as it hurdles the definitive treatment of the $\mathrm{PNH}$, an allogenic bone marrow transplantation. but should be reserved for patients with suboptimal response to eculizumab ${ }^{(6)}$

\section{Conclusion}

As definitive treatment is now available for the PNH patient, it is important to notice the unusual presentation of the disease and it helps for physician to be broad minded and have high suspicious of the rare presentation of the $\mathrm{PNH}$, so early diagnosis and timely treatment of these patients is lifesaving.

Data from this registry should help define the natural history of PNH since the introduction of therapy that inhibits terminal complement. ${ }^{(7)}$ In the coming years, novel inhibitors of the alternative pathway of complement ${ }^{(8-9)}$ and complement inhibitors with extended half-lives are likely to further improve quality of life for PNH patients.

\section{References}

1. R.L. Rietschel, C.W. Lewis, R.A. Simmons, et al. Skin lesions in paroxysmal nocturnal haemoglobinuria Arch Dermatol, 114 (1978), pp. 560-563.

2. J.M. White, K. Watson, R. Arya, et al. Haemorrhagic bullae in a case of paroxysmal nocturnal haemoglobinuria Clin Exp Dermatol, 28 (2003), pp. 504-505. 
3. C. Cholez, J.L. Schmutz, C. Hulin, et al. Cutaneous necrosis during paroxysmal nocturnal haemoglobinuria: role of parvovirus B19?JEur Acad Dermatol Venereol, 19 (2005), pp. 381-382

4. J. Alves, H. Barreiros, D. Matos, R. Coelho Cutaneous thrombosis related to paroxysmal nocturnal haemoglobinuria: clinical report Dermatol Online J, 18 (2012), p. 13

5. O. Salim, O.K. Yücel, et al. Cutaneous thrombosis as the presenting finding of paroxysmal nocturnal haemoglobinuriaBr $\mathbf{J}$ Haematol, 171 (2015), p. 296. [Epub 2015 Jul 23]

6. R.A. Brodsky Paroxysmal nocturnal haemoglobinuria Blood, 124 (2014), pp. 28042811

7. Schrezenmeier H, Muus P, Socié G, etal. $B a$ seline characteristics and disease burden in patients in the international paroxysmal nocturnal haemoglobinuria registry. Haematologica 2014.

8. Lindorfer MA, Pawluczkowycz AW, et al. A novel approach to preventing the haemolysis of paroxysmal nocturnal haemoglobinuria: both complement-mediated cytolysis and C3 deposition are blocked by a monoclonal antibody specific for the alternative pathway of complement. Blood 2010;115(11):22832291.

9. Risitano AM, Notaro R, Pascariello C, et al. The complement receptor 2/factor $H$ fusion protein TT30 protects paroxysmal nocturnal haemoglobinuria erythrocytes from complement-mediated haemolysis and C3 fragment. Blood 2012;119(26):6307-6316. 